Benchmarks

\title{
The fluorescent dyes TO-PRO-3 and TOTO-3 iodide allow detection of microbial cells in soil samples without interference from background fluorescence
}

Ruth Henneberger ${ }^{1,}$, Debra Birch ${ }^{2}$, Peter Bergquist ${ }^{3}$, Malcolm Walter $^{4}$, and Roberto P. Anitori ${ }^{1, * *}$

${ }^{1}$ Department of Chemistry and Biomolecular Sciences, ${ }^{2}$ Department of Biology, Macquarie University, Sydney, NSW 2109, Australia, ${ }^{3}$ Department of Molecular Medicine and Pathology, University of Auckland School of Medicine, Auckland, New Zealand, and ${ }^{4}$ Australian Centre for Astrobiology, University of New South Wales, Sydney, 2052, Australia

BioTechniques 51:190-192 (September 2011) doi 10.2144/000113736

Keywords: FISH; fluorescence; soil; microbes; TO-PRO-3; TOTO-3

Supplementary material for this article is available at www.BioTechniques.com/article/113736.

"R.H. current address is Institute for Biogeochemistry and Pollutant Dynamics, Swiss Federal Institute of Technology ETH, Universitätstrasse 16, ETH-Zentrum, CH-8092, Zürich, Switzerland.

"* R.P.A. current address is Division of Environmental and Biomolecular Systems, Department of Science and Engineering, Oregon Health and Science University, Beaverton, OR 97006, USA.

Visualization of microorganisms in soils and sediments using fluorescent dyes is a common method in microbial ecology studies, but is often hampered by strong nonspecific background fluorescence that can mask genuine cellular signals. The cyanine nucleic acid binding dyes TO-PRO-3 and TOTO-3 iodide enabled a clear detection of microbial cells in a mineral soil, while nonspecific background was greatly reduced compared with commonly used dyes. When used as counterstains for fluorescence in situ hybridization (FISH), both cyanine dyes allowed identification of microbial cells despite strong background from nonspecifically bound probes. TO-PRO-3 and TOTO-3 are easy to use and represent superior alternatives for detecting microorganisms in soil environments.

Fluorescence microscopy with nucleic acid-specific dyes, such as 4',6-diamidino2-phenylindole (DAPI) or acridine orange, is used widely to visualize microbial cells in environmental samples independently of their physiological state or phylogenetic placement (1). However, inefficient staining, nonspecific binding to sample components, and autofluorescence of mineral particles under UV excitation often interfere with efficient cell detection, in particular in soil and sediment samples (2). Novel high-affinity stains, such as SYBR Green, Sytox, or Syto dyes, promise better signal-to-background ratios due to their increased fluorescence when bound to DNA (3-5). Nevertheless, the general issue of nonspecific background fluorescence remains problematic for many sample types. Excitation of nucleic acid-specific dyes with monochromatic light (e.g., in confocal laser scanning microscopy; CLSM) can reduce such background fluorescence, and CLSM is now often used for direct cell counts and fluorescence in situ hybridization (FISH) applications in environmental studies $(2,6)$. However, many commonly used dyes require excitation in the UV range, for which confocal microscopes often are not properly equipped. In contrast, the monomeric TO-PRO-3 iodide (TP3; 642/661 nm excitation/emission peaks) and the dimeric TOTO-3 iodide (TT3; $642 / 660 \mathrm{~nm}$ ) can be excited with a standard CLSM He-Ne 633 nm laser. While not assessed in the present study, they can also be used with light microscopes that are equipped for detection of red fluorescence $(630-700 \mathrm{~nm})$. These cyanine dyes display a high affinity for double-stranded nucleic acids in fixed cells, fluorescing strongly as DNA-dye complexes but only weakly prior to DNA intercalation. TP3 is widely applied to stain DNA in eukaryotic cells (e.g., for flow cytometry, DNA quantification, or as a FISH counterstain) (7-9). Even though reports on using TP3 to stain prokaryotic cells are limited to flow cytometry of pure cultures $(10,11)$, the weak fluorescence of unbound and nonspecifically bound dye molecules and the lower levels of autofluorescence with high wavelength excitation make TP3 and TT3 promising alternatives for the visualization of microbial cells in natural ecosystems. Here we present our results on the use of these dyes as both general nucleic acid stains for detecting microorganisms in mineral-rich soils and as FISH counterstains.

Initially, pure microbial cultures were stained with both dyes to evaluate optimal dye concentrations and staining conditions. Samples were analyzed by CLSM, and staining efficiency was determined by visual comparison of signal intensities and numbers of stained cells (see the Supplementary materials). Fixed cells of pure cultures (Escherichia coli, Sulfobacillus sp., Sulfolobus metallicus, and the SM1 euryarchaeon) (12) showed clear fluorescent signals when stained for $15 \mathrm{~min}$ with $1-5$ $\mu \mathrm{M}$ TP 3 or TT3 in $0.01 \mathrm{M}$ Tris- $\mathrm{HCl}, \mathrm{pH}$ 7.2. Nonspecific background fluorescence was not detected, and signal intensities increased with increasing dye concentrations up to 4-5 $\mu \mathrm{M}$ (see Supplementary Figure S1). In general, signal intensity for TT3 appeared slightly lower compared with TP3, and addition of SDS to the staining buffer resulted in slightly 


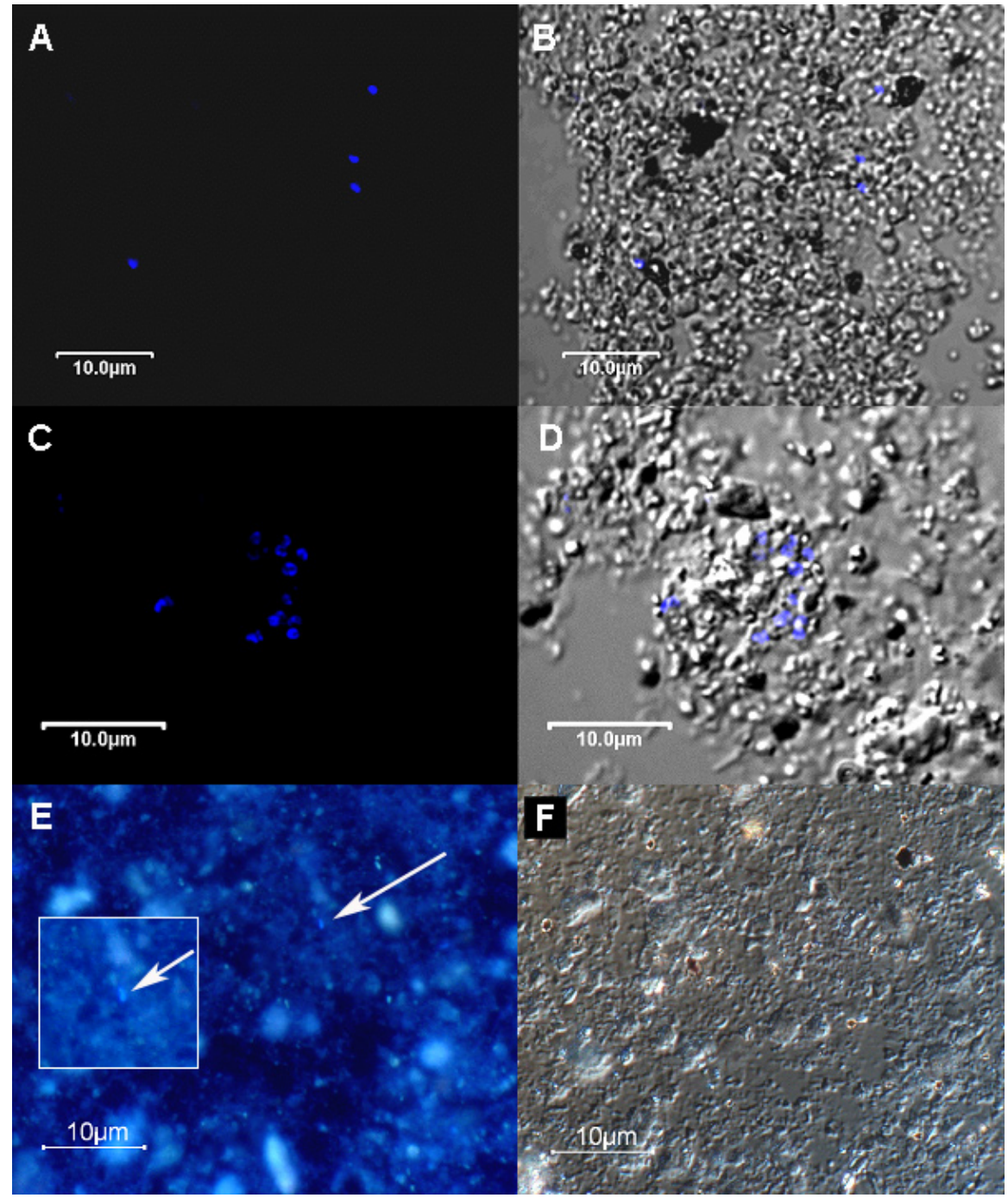

Figure 1. Application of TP3 for the visualization of microbial cells in mineral-rich soil. (A-D) CLSM micrographs of samples stained with TP3, small, coccoid cells visible. (A and B) Same field of view shown; (A) TP3 fluorescence; (B) fluorescence and DIC overlay. (C and D) Same field of view shown; (C) TP3 fluorescence; (D) fluorescence and DIC overlay. (E and F) Epifluorescence micrograph, same field of view shown; (E) DAPI, putative cell indicated by arrow (magnification in insert); (F) DIC and DAPI overlay. Scale bars, $10.0 \mu \mathrm{m}$.

reduced fluorescence. On the other hand, TP3 showed higher sensitivity to photobleaching, which agrees with published results for eukaryotic cells $(13,14)$.

The suitability of TT3 and TP3 for detecting cells within mineral-rich samples subsequently was tested using hydrothermally degraded soils with small particlesizes and high clay contents (from Mt. Hood, Oregon, USA) (15). When stained with DAPI or SYBR Green I (data not shown for latter), these soils showed strong nonspecific background fluorescence of mineral particles with cell-like sizes and shapes. To evaluate the suitability of TP3 and TT3, sterile soil samples were spiked with bacterial and archaeal cells prior to staining (see the Supplementary material). After incubation for $1 \mathrm{~h}$, fluorescent cells were clearly visible within and distinguishable from mineral particles, while nonspecific background fluorescence was absent (data not shown). Stained, unspiked control samples did not display any signals or background fluorescence, indicating that signals observed in the spiked samples corresponded to cells and not mineral particles. Despite its higher sensitivity to photobleaching, TP3 showed superior signal intensities, allowing improved detection of cells compared with TT3, and was subsequently used to stain unspiked background fluorescence was very low, and endogenous cells were clearly visible and distinguishable from sample particles. Small, single coccoid cells (Figure 1, A and B) and cell clusters associated with Mt. Hood samples. Again, nonspecific mineral structures (Figure 1, C and D) were identified, whereas staining with DAPI resulted in nonspecific background fluorescence, masking any cellular signals present (Figure 1, E and F).

Both dyes were also tested as DNA counterstains for microbial FISH. TP3 and TT3 signals were clearly visible in bacterial and archaeal cultures hybridized with domain-specific probes (data not shown). The counterstaining did not influence FISH signals, but laser excitation of the probes resulted in photobleaching and reduced signal intensities, in particular for TP3. In spiked and unspiked Mt. Hood samples, cells were identified by positive TT3 staining after hybridization and counterstaining, despite very strong background fluorescence of nonspecifically bound FISH probes and autofluorescence of minerals that masked cellular FISH signals. Furthermore, it was possible, using image overlay, to detect putative archaeal FISH signals that would have been overlooked without TT3 counterstaining (Figure 2).

To the authors' knowledge, application of TP3 and TT3 to directly visualize prokaryotic cells in environmental samples has not been reported previously. Our experiments show that TP3 and TT3 are promising for the detection of microorganisms in soils with high particle loads. The weak fluorescence of unbound or nonspecifically bound dye molecules and the use of high-wavelength monochromatic excitation light significantly reduced nonspecific background. Samples can be stained directly with a simple protocol, while aggressive pretreatment is not required (16). These properties make TP3 and TT3 superior to other commonly used dyes for visualizing microorganisms in complex samples such as soils and, presumably, sediments. The dyes also show potential to be applied in FISH applications in which the number of mineral particles nonspecifically stained by the FISH probe are not excessively high, and far-red excitation and emission will allow combination with many commonly used fluorochromes with shorter excitation maxima.

\section{Acknowledgments}

R.H. was funded by Macquarie University (Postgraduate Research Fund and an International Travel Scholarship). We are grateful to the Mazamas Research Committee for financially supporting the sample collection, and Dana Rogoff, Emily Ohlin, Tom Bennett, Steve Boyer, Bob McGown, and Dave Jordan for assistance 


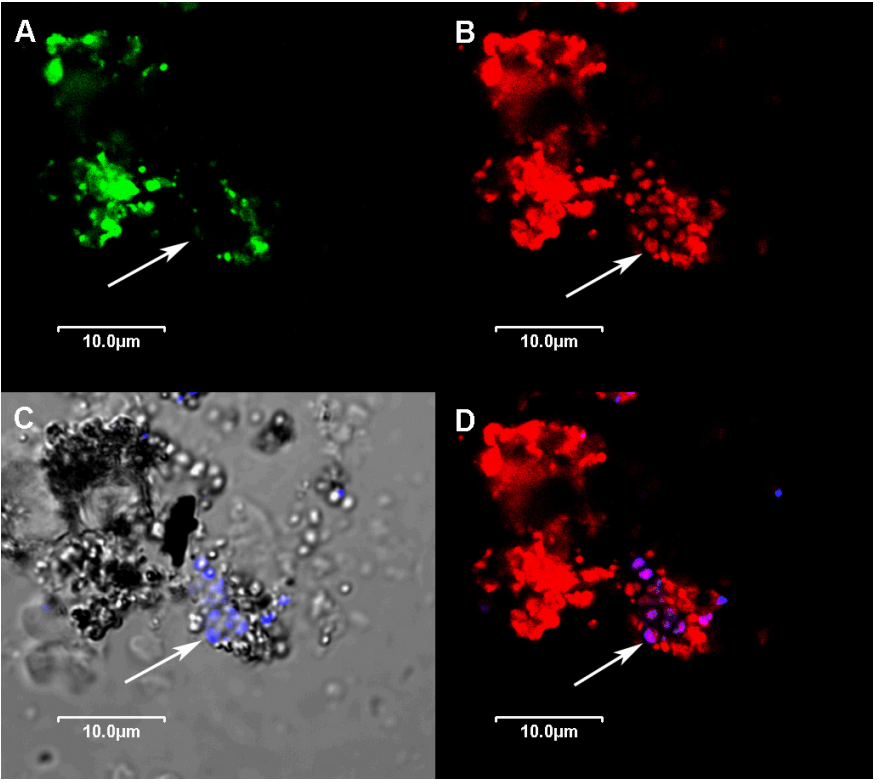

Figure 2. Application of TT3 as a FISH counterstain in mineral-rich soil. CLSM micrograph labeled with EUB-Mix (Alexa488, green), ARCH-Mix (Cy3, red), and TT3; same field of view shown; arrow pointing to a group of cells. (A) FISH (EUB-Mix). (B) FISH (ARCH-Mix). (C) Overlay of DIC and TT3 fluorescence. (D) Overlay of FISH (ARCH-Mix) and TT3 fluorescence, colocalization of ARCH-Mix and TT3 visible in purple. Scale bars, $10.0 \mu \mathrm{m}$.

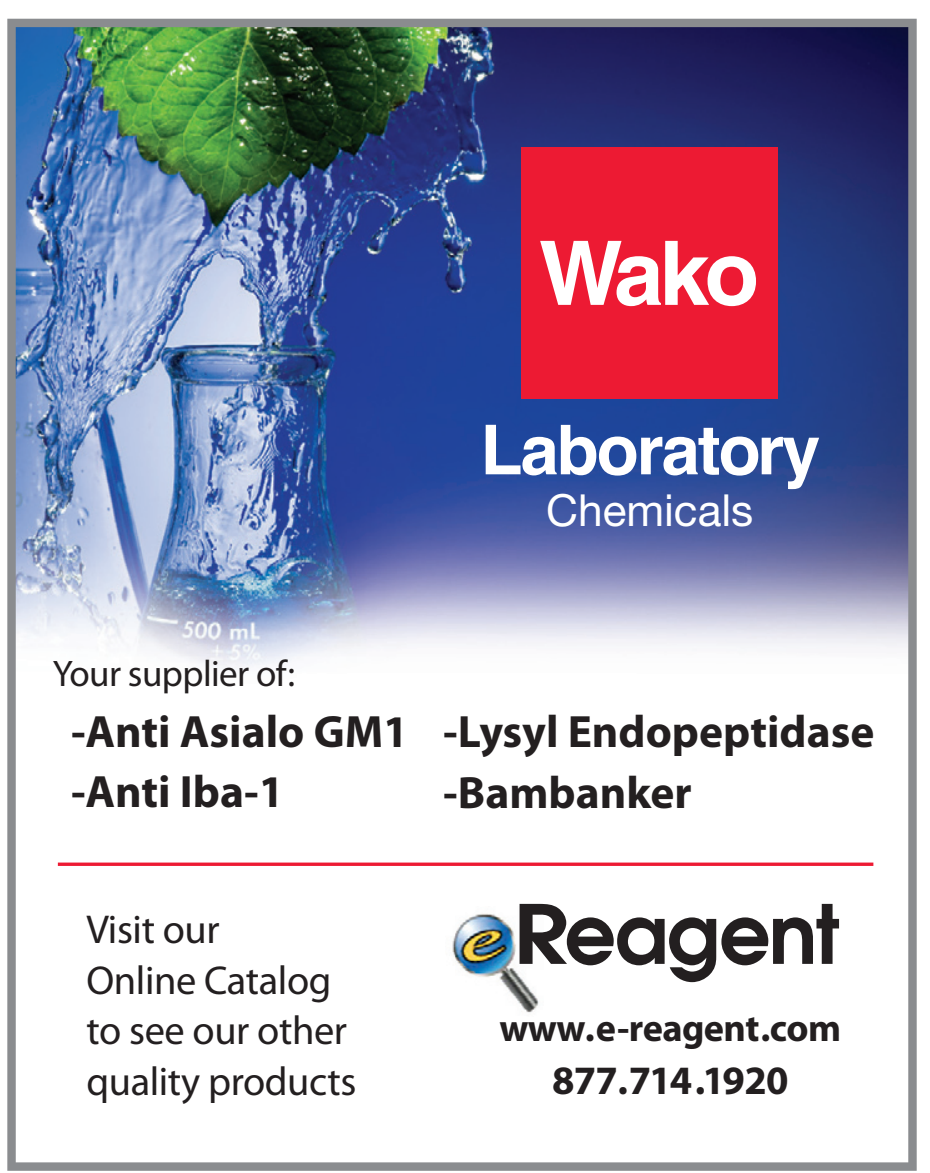

during sample collection, and Russell Field for particle size analysis.

\section{Competing interests}

The authors declare no competing interests.

\section{References}

1. Kepner, R.L. and J.R. Pratt. 1994. Use of fluorochromes for direct enumeration of total bacteria in environmental samples: past and present. Microbiol. Rev. 58:603-615.

2. Li, Y., W.A. Dick, and O.H. Tuovinen. 2004. Fluorescence microscopy for visualization of soil microorganisms-a review. Biol. Fertil. Soils 39:301-311.

3. Klauth, P., R. Wilhelm, E. Klumpp, L. Poschen, and J. Groeneweg. 2004. Enumeration of soil bacteria with the green fluorescent nucleic acid dye Sytox green in the presence of soil particles. J. Microbiol. Methods 59:189-198.

4. Lebaron, P., N. Parthuisot, and P. Catala. 1998. Comparison of blue nucleic acid dyes for flow cytometric enumeration of bacteria in aquatic systems. Appl. Environ. Microbiol. 64:1725-1730.

5. Weinbauer, M.G., C. Beckman, and M.G. Höfle. 1998. Utility of green fluorescent nucleic acid dyes and aluminum oxide membrane filters for rapid epifluorescence enumeration of soil and sediment bacteria. Appl. Environ. Microbiol. 64:5000-5003.

6. Amann, R.I., W. Ludwig, and K.H. Schleifer. 1995. Phylogenetic identification and in situ detection of individual microbial cells without cultivation. Microbiol. Rev. 59:143-169.

7. Bink, K., A. Walch, A. Feuchtinger, H. Eisenmann, P. Hutzler, H. Höfler, and M. Werner. 2001. TO-PRO-3 is an optimal fluorescent dye for nuclear counterstaining in dual-colour FISH on paraffin section. Histochem. Cell Biol. 115:293-299.

8. Ploeger, L.S., H.F.J. Dullens, A. Huisman, and P.J. van Diest. 2007. Fluorescent stains for quantification of DNA by confocal laser scanning microscopy in 3-D. Biotech. Histochem. 82:1-7.

9. Tavecchio, M., M. Simone, S. Bernasconi, G. Tognon, G. Mazzini, and E. Erbe. 2008. Multi-parameter flow cytometric cell cycle analysis using TO-PRO-3 iodide (TP3): detailed protocols. Acta Histochem. 110:232-244

10. Novo, D.J., N.G. Permutter, R.H. Hunt, and H.M. Shapiro. 2000. Multiparameter flow cytometric analysis of antibiotic effects on membrane potential membrane permeability, and bacterial counts of Staphylococcus aureus and Micrococcus luteus. Antimicrob. Agents Chemother. 44:827-834.

11. Shapiro, H.M. 2001. Multiparameter flow cytometry of bacteria: implications for diagnostics and therapeutics. Cytometry 43:223-226.

12. Rudolph, C., G. Wanner, R. Huber. 2001. Natural communities of novel archaea and bacteria growing in cold sulfurous springs with a string-ofpearls-like morphology. Appl. Environ. Microbio. 67:2336-2344.

13. Beisker, W., E.M. Weller-Mewe, and M. Nüsse. 1999. Fluorescence enhancement of DNA-bound TO-PRO-3 by incorporation of bromodeoxyuridine to monitor cell cycle kinetics. Cytometry 1:221-229.

14. Matsusaki, T., T. Suzuki, K. Fujikura, and K. Takata. 1997. Nuclear staining for laser confocal microscopy. Acta Histochem. Cytochem. 30:309-314.

15. Henneberger, R.M., M.R. Walter, and R.P. Anitori. 2006. Extraction of DNA from acidic, hydrothermally modified volcanic soils. Environ. Chem. 3:100-104.

16. Morono, Y., T. Terada, N. Masui, and F. Inagaki. 2009. Discriminative detection and enumeration of microbial life in marine subsurface sediments. ISME J. 3:503-511.

Received 12 May 2011; accepted 12 August 2011.

Address correspondence to Roberto P. Anitori, Division of Environmental and Biomolecular Systems, Department of Science and Engineering, Oregon Health and Science University, Beaverton, OR 97006, USA.e-mail: anitorir@ebs.ogi.edu

To purchase reprints of this article, contact: biotechniques@fosterprinting.com 